\title{
Co-transplantation of bone marrow mesenchymal stem cells and monocytes in the brain stem to repair the facial nerve axotomy
}

\author{
Li Wu, ${ }^{1}$ Dan Han, ${ }^{1}$ Jie Jiang, ${ }^{1}$ Xiaojie Xie, ${ }^{1}$ Xunran Zhao, ${ }^{1}$ Tengfei Ke, ${ }^{2}$ Wen Zhao, ${ }^{1}$ Liu Liu, ${ }^{3}$ Wei Zhao \\ ${ }^{1}$ Department of Medical Imaging, First Affiliated Hospital of Kunming Medical University, Kunming \\ ${ }^{2}$ Department of Medical Imaging, Third Affiliated Hospital of Kunming Medical University, Kunming \\ ${ }^{3}$ Department of Plastic Surgery, First Affiliated Hospital of Kunming Medical University, Kunming, China
}



\begin{abstract}
After the facial nerve axotomy (FNA), the distal end of the axon would gradually decay and disappear. Accumulated evidence shows that transplantation of bone marrow mesenchymal stem cells (BMSCs) reveals potential in the treatment of nervous system diseases or injuries. This study is aimed at investigating the therapeutic effects of co-transplantation of BMSCs and monocytes in FNA. We found that co-culture significantly elevated the CD4+/CD8+ ratio and CD4+ CD25+ T cell proportion compared with monocytes transplantation, and enhanced the differentiation of BMSCs into neurons. After the cell transplantation, the lowest apoptosis in the facial nerve nucleus was found in the co-transplantation group 2 (BMSCs:monocytes= 1:30). Moreover, the lowest expression levels of pro-inflammatory cytokines and the highest expression levels of anti-inflammatory cytokines were observed in the co-transplantation group 2 (BMSCs: monocytes $=1: 30$ ). The highest expression levels of protein in the JAK/STAT6 pathway and the SDF-1/CXCR4 axis were found in the co-transplantation group 2. BMSC/monocyte co-transplantation significantly improves the microenvironment in the facial nerve nucleus in FNA rats; therefore these findings suggest that it could promote the anti-/pro-inflammatory balance shift towards the anti-inflammatory microenvironment, alleviating survival conditions for BMSCs, regulating BMSC the chemotaxis homing, differentiation, and the section of BMSCs, and finally reducing the neuronal apoptosis. These findings might provide essential evidence for the in-hospital treatment of FNA with co-transplantation of BMSCs and monocytes.
\end{abstract}

Key words: Facial nerve axotomy (FNA); bone marrow mesenchymal stem cells (BMSCs); monocytes; cotransplantation; microenvironment.

Correspondence: Liu Liu, Department of Plastic Surgery, the First Affiliated Hospital of Kunming Medical University, No. 295, Xichang Road, Kunming 650032, Yunnan, China. Tel. +86.18887112038. E-mail: liuliuwuli@126.com

Contributions: LW, DH contributed equally to this work. LW, WZ, JJ, XX, WeiZ, XZ, experiments performing; LW, data analysis, manuscript drafting; LL, study design, manuscript editing. All authors read and approved the final manuscript. All the authors have read and approved the final version of the manuscript and agreed to be accountable for all aspects of the work.

Conflict of interest: The authors declare that they have no conflict of interests.

Funding: This work was supported by the National Natural Science Foundation of China Grant (No. 81460298) and by the Yunnan Provincial Science and Technology Department-Kunming Medical University Applied Basic Research Joint Special Fund Grant (No. 2015FB038).

Availability of data and materials: Data sets used and/or analyzed while carrying out this study are available from the corresponding author upon reasonable request.

Ethical Approval: The experiment was conducted in accordance with the International Association of Veterinary Editors' Consensus on Authors' Guidelines on Animal Ethics and Welfare and with local and national regulations.

Patient consent for publication: Not applicable. 


\section{Introduction}

Facial nerve axotomy (FNA) would result in a functional disability of facial expression muscles, which severely influences the life quality of patients. ${ }^{1}$ At present, the facial nerve in situ restoration is the primary treatment option for in-hospital FNA, with satisfactory functional recovery of facial expression muscles. However, compared with pre-operation, some patients undergoing restoration might feel an obvious difference in nerve function. The restoration and reconstruction of facial nerves, therefore, still remain a difficult issue in the field of plastic surgery and neurology. In recent years, transplantation of bone marrow mesenchymal stem cells (BMSCs) has been widely used in hospital for the treatment of nervous system diseases or injuries. Numerous studies have shown that the transplanted BMSCs would differentiate into neuron-like cells or neuronal stem cells in the microenvironment of the central nervous system (CNS). When transplanted, the exogenous BMSCs could recognize the chemokines and migrate to the lesion sites, which adhere to the blood vessels around the lesion, eventually colonized..$^{2,3}$ Moreover, these transplanted cells could secrete various kinds of neurotrophic factors to promote the neurogenic process. However, the survival rate of transplanted BMSCs is relatively low due to the injury-induced proinflammatory microenvironment, hypoxia, and hypoperfusion. ${ }^{4}$ After the facial nerve axonal injury, the damage signal from the neural broken ends would be delivered along the proximal axon into the facial motoneurons (FMNs), leading to the proinflammatory microenvironment in the facial nerve nucleus.

Due to the proinflammatory status, chemokines could be generated to recruit the peripheral $\mathrm{T}$ cells and autologous stem cells into the facial nucleus. As the subsets of $\mathrm{T}$ lymphocytes, the CD4+CD25+regulatory $\mathrm{T}$ cells (Treg) play important roles in maintaining the balance of proinflammatory microenvironment in the facial nerve nucleus, as well as in the protection of the apoptotic neurons. ${ }^{5-8}$ Autologous stem cells can differentiate into neural cells to replace or sustain the damaged FMNs. However, the proinflammatory microenvironment in the facial nerve nucleus jeopardizes the survival of the stem cells, and these autologous stem cells cannot fully make up for the loss of FMNs. Studies have shown that BMSCs reveals a potential in the immune suppressive effects, which could inhibit the proliferation of $\mathrm{T}$ cells, regulate the immune responses, and increase the proportion of CD4+ CD25+ Treg cells in the in vitro mixed lymphocyte culture. ${ }^{9,10}$

In this study, the SD rat-derived BMSCs were co-cultured with the spleen monocytes, which were co-transplanted into the facial nerve nucleus of the brain stem in the rats that suffered from unilateral FNA. The proportion of CD4+ CD25+ Treg cells, the proinflammatory microenvironment, and the generation and secretion of neurotrophic factors were investigated.

\section{Materials and Methods}

\section{Animals and cell lines}

Six to eight-week-old male SD rats (SPF grade) weighing 180$250 \mathrm{~g}$ were purchased from Dashou Experimental Animal Co., Ltd. (License No. SCXK Chuan 2008-24; Chengdu, Sichuan, China). Primary monocytes were isolated from the spleen of the rats. All the animal experimental procedures were approved by the Ethics Committee of the Kunming Medical University. BMSCs expressing GFP (GFP-BMSCs) were purchased from the Cyagen Biosciences (Lot No. 121212L01; Guangzhou, Guangdong, China).

\section{Co-culture of SPIO-GFP-BMSCs and monocytes}

Primary monocytes were isolated and cultured from the spleen in the SD rats (the culture of primary monocytes is shown in Supplement 1), with the cell isolation kit (Solarbio, Beijing, China). GFP-BMSCs were planted and cultured on the 24-well plate (each well contained a disk), at the density of $1 \times 10^{4}$ cells/well. These cells were cultured with complete culture medium containing superparamagnetic iron oxide (SPIO) (with the final iron concentration of $75 \mathrm{mg} / \mathrm{mL}$; Sigma) in the $37^{\circ} \mathrm{C}, 5 \% \mathrm{CO}_{2}$ incubator for $24 \mathrm{~h}$. For the co-culture of SPIO-GFP-BMSCs and monocytes, SPIO-GFP-BMSCs were first planted onto the lower layer of the Transwell cell culture plate (each well contained a disk; Corning, New York, NY, USA). Monocytes were seeded in the upper chamber. According to the pre-experiments, the co-culture ratios of SPIO-GFP-BMSCs and monocytes were set at 30:1 (co-culture group 1) and 1:30 (co-culture group 2), respectively (The selection of ratio is shown in Supplement 2). Cells were cultured with the phytohemagglutinin (PHA) (Sigma) which contained BMSC complete medium, i.e., DMEM/F12 medium (Gibco) containing 10\% fetal bovine serum (FBS), 1\% streptomycin, and $1 \%$ L-glutamine. The culture medium was replaced every $48 \mathrm{~h}$.

\section{Protein array analysis}

Protein contents in the SPIO-GFP-BMSCs, as well as in the culture supernatant of the co-culture (both co-culture groups 1 and 2) were investigated by means of protein array analysis. Briefly, the SPIO-GFP-BMSCs and the culture supernatant in the co-culture were separated and collected by centrifugation respectively. Cell lysate was obtained with the lysis. Protein array analysis was performed with the Raybiotech antibody array kit (Lot No. AARCYT-G2; Raybiotech, Norcross, GA, USA), according to the manufacturer's instructions. Briefly, the chip well was first blocked with $100 \mathrm{~mL} 1 \times$ blocking solution at room temperature for $1 \mathrm{~h}$. For detection, $100 \mathrm{~mL}$ sample $(300 \mathrm{mg} / \mathrm{mL})$ was added into each well and the chip was incubated at $4^{\circ} \mathrm{C}$ overnight. After washing with the Thermo Scientific Wellwash Versa chip washing machine (Thermo Scientific, Waltham, MA, USA), $70 \mathrm{~mL}$ biotin-labeled antibody was added into each well, which was incubated at room temperature for $2 \mathrm{~h}$. After washing, $70 \mathrm{~mL}$ fluorescent agent-streptavidin was added into each well, then sealed and incubated in the dark at room temperature for $2 \mathrm{~h}$. The fluorescence was detected with the GenePix 4000B Microarray Scanner (Molecular Devices, Sunnyvale, CA, USA).

\section{Flow cytometry}

Flow cytometry was performed to investigate the proportion of CD4+ CD25+ T cells and the CD4+/CD8+ ratio in the monocytes of the co-culture. On day 7 of co-culture, the medium from the upper chamber was collected and diluted with equal volume of phosphate buffer saline (PBS). The mixture was centrifuged at $1000 \mathrm{rpm}$ for $5 \mathrm{~min}$ and cells in the precipitation were fixed with pre-cooled $75 \%$ alcohol at $4{ }^{\circ} \mathrm{C}$ for $30 \mathrm{~min}$. After centrifugation at $1000 \mathrm{rpm}$ for $5 \mathrm{~min}$, cells were blocked with 1\% BSA (Aladdin, Shanghai, China) at room temperature for $1 \mathrm{~h}$. After centrifugation at $1000 \mathrm{rpm}$ for $5 \mathrm{~min}$, cells were incubated with CD4\&CD8 and CD4\&CD25 antibody working solution (both from eBioscience, San Diego, CA, USA), respectively, in the dark for $30 \mathrm{~min}$. After centrifugation at $1000 \mathrm{rpm}$ for $5 \mathrm{~min}$, cells were re-suspended with $800 \mathrm{~mL}$ phosphate buffer saline (PBS) $(0.01 \mathrm{M})$ and detected by flow cytometry in $6 \mathrm{~h}$.

\section{Immunofluorescence staining}

The effects of co-culture on the differentiation potential of SPIO-GFP-BMSCs were investigated by immunofluorescence 
staining. Briefly, after $96 \mathrm{~h}$ co-culture, the disk in the chamber on which the SPIO-GFP-BMSCs grew was treated with $0.5 \mathrm{~mL} \mathrm{3 \%}$ peroxide in the dark for $30 \mathrm{~min}$. After washing with $0.1 \mathrm{M}$ PBS, cells were blocked with $0.5 \mathrm{ml} 5 \%$ goat serum at room temperature for $1 \mathrm{~h}$. Then, cells were incubated with mouse-anti-rat anti-NeuN primary antibody (1:500 dilution; Millipore, Massachusetts, MA, USA) and mouse-anti-rat anti-NF-200 primary antibody (1:500 dilution; Millipore), respectively, at $4^{\circ} \mathrm{C}$ overnight. Subsequently, cells were incubated with DyLight conjugated goat anti-mouse secondary antibody (1:2000 dilution; Jackson Immuno-Research, West Grove, PA, USA) in the dark at room temperature for $2 \mathrm{~h}$. After sealing, the fluorescence was observed under a fluorescence microscope (Olympus, Tokyo, Japan).

\section{Model establishment and grouping}

Rat models of unilateral FNA at stylomastoid foramen were established, according to previously published protocols. ${ }^{11}$ Briefly, the rats were anesthetized by $10 \%$ chloral hydrate $(3 \mathrm{mg} / \mathrm{kg}$ body weight). No signs of peritonitis were founded after the administration of $10 \%$ chloral hydrate. After the iodophor disinfection and skin preparation, a $1 \mathrm{~cm}$ vertical incision was made along the retroauricular groove to expose the facial nerve trunk. The stylomastoid foramen was located along the nerve trunk. The facial nerve, and more precisely, the facial nerve trunk and the facial nerve behind the ear were divided into two branches. At the stylomastoid foramen, the facial nerve was gently separated and pulled outside for 2 $\mathrm{mm}$ with an ophthalmic tweezer. Then the facial nerve was cut by ophthalmic shears, resulting in FNA at high position, and an approximately $2 \mathrm{~mm}$ fragment was cut off on the distal end. The skin incision was then sutured up. For the sham operation, the skin incision was made and the facial nerve was exposed up to the stylomastoid foramen. No cut was made on the facial nerve. After surgery, the rat models were re-warmed in the incubator, and then returned to the cage with regular feeding. The facial paralysis was observed every $24 \mathrm{~h}$. According to the House-Brackmann grading system (BHGS), these models were all classified as full paralysis, with regard to the stationary vibrissae on the surgical side, with no eye-blink reflex.

These rats were divided into 6 groups. The negative control (sham) group, in which the rats without facial nerve cut were treated with SPIO and PBS; the positive control (NS) group, in which the rat models of FNA were only treated with SPIO and PBS; the mononuclear transplantation group, in which the rats with FNA were transplanted with SPIO-labeled monocytes alone; the BMSC transplantation group, in which the rats with FNA were transplanted with the SPIO-GFP-BMSCs alone; the co-transplantation group 1 , in which the rats with FNA were co-transplanted with SPIOGFP-BMSCs and monocytes (BMSCs:monocytes = 30:1); and the co-transplantation group 2, in which the rats with FNA were cotransplanted with SPIO-GFP-BMSCs and monocytes (BMSCs:monocytes $=1: 30$ ).

\section{Cell transplantation}

For cell transplantation, the rat was anesthetized by $10 \%$ chloral hydrate ( $3 \mathrm{mg} / \mathrm{kg}$ body weight) and mounted on the stereotaxic apparatus. The hair on the head was cut off. After the iodophor disinfection, a $2 \mathrm{~cm}$ skin incision was made along with the sagittal suture. The subcutaneous tissue was separated, and the fascia was cleared with hydrogen peroxide. According to the stereotactic map of rat brain, the center of facial nerve nucleus was located based on the following coordinates, $11.3 \mathrm{~mm}$ behind the bregma, $2.5 \mathrm{~mm}$ beside the sagittal suture, and $8 \mathrm{~mm}$ beneath the skull. A small hole ( $2 \mathrm{~mm}$ in diameter) was made by the electric drill, at $11.3 \mathrm{~mm}$ behind the bregma and $2.5 \mathrm{~mm}$ beside the sagittal suture. A $10 \mathrm{~mL}$ microinjection needle (containing $1 \times 10^{6}$ cells in $5 \mathrm{~mL}$ suspension) was slowly inserted into the center of the facial nerve nucleus. The cell suspension was injected at the rate of $1 \mathrm{ml} / 30 \mathrm{~s}$, for $2.5 \mathrm{~min}$. Then the needle was slowly pulled out and the skin was sutured up. For the sham group, negative control animals were injected with 4 $\mathrm{mL}$ PBS and $1 \mathrm{~mL}$ SPIO $(75 \mathrm{mg} / \mathrm{mL})$. For the NS group, model animals were injected with $4 \mathrm{mLPBS}$ and $1 \mathrm{~mL}$ SPIO $(75 \mathrm{mg} / \mathrm{mL})$. After cell transplantation, the rat models were re-warmed in the incubator, and then returned into the cage. No signs of peritonitis were founded after the administration of $10 \%$ chloral hydrate.

For the sample preparation, on days $0,3,7,14,21$, and 28 posttransplantation, the animals were euthanized by cervical dislocation. Brain tissue samples were collected and subjected to the following histological staining, western blot analysis, and quantitative real-time PCR detection.

\section{Histological staining}

The extracted brain tissue was fixed with $4 \%$ paraformaldehyde. The facial nerve nucleus on the brain stem side (at which point the trigeminal nerve exited from the brain stem) was harvested (about $5 \mathrm{~mm}$ in thickness). After dehydration, transparency, and embedding, the tissue sample was cut into $5 \mathrm{~mm}$ serial sections. Following the routine of paraffinized and hydration, sections were blocked with $5 \%$ goat serum at room temperature for $60 \mathrm{~min}$, and then incubated with mouse-anti-rat anti-NeuN primary antibody (1:100 dilution; Millipore, Bedford, MA, USA) and mouse-anti-rat NF200 primary antibody (1:200 dilution; Abcam, Cambridge, UK) at $4^{\circ} \mathrm{C}$ overnight. After washing with PBST, sections were incubated with PV9000 I and PV9000 II reagents (both from ZSGB-BIO, Beijing, China), subsequently, at $37^{\circ} \mathrm{C}$, for $30 \mathrm{~min}$. DAB colorization was conducted in dark at room temperature for $5 \mathrm{~min}$. After dehydration with alcohol gradients and transparency with xylene, sections were sealed and observed under microscope (Olympus).

\section{TUNEL staining}

Cellular apoptosis was detected by terminal deoxynucleotidyl transferase (TdT)-mediated dUTP nick-end labeling (TUNEL) assay kit (Boster, Wuhan, Hubei, China), according to the manufacturer's instructions. Briefly, after washing with PBS, cells were incubated with $20 \mathrm{mg} / \mathrm{ml}$ protease $\mathrm{K}$ without DNase at $37^{\circ} \mathrm{C}$ for 5 min. Then they were labeled with biotin in $50 \mathrm{ml}$ buffer in the dark at $37^{\circ} \mathrm{C}$ for $60 \mathrm{~min}$. After washing, $0.2 \mathrm{ml}$ stop solution was added to incubate the cells at room temperature for $10 \mathrm{~min}$. Then, cells were treated with $50 \mathrm{ml}$ Streptavidin-HRP working solution in the dark at room temperature for $30 \mathrm{~min}$. Color development was performed with the DAB solution at room temperature for $10 \mathrm{~min}$. Cellular apoptosis was observed under the microscope (Olympus).

\section{Quantitative real-time PCR}

Total RNA was extracted with the TRIZOL agent. The cDNA template was obtained by reverse transcription. Quantitative realtime PCR was performed with the SYBR Green master mix kit (Kapa Biosystems, Woburn, MA, USA) on the ABI 7300 PCR machine (ABI, Foster City, CA, USA). PCR primer sequences were as follows: IL4, forward 5'-TTGAACCAGGTCACAGAA3' and reverse 5'-GTTCCTTGTTGCCGTAAG-3'; SDF1, forward 5'-AAGCAACAACAGACAAGT-3' and reverse 5'CACATCTTGAGCCTCTTG-3'; and BDNF, 5'-CTACCCAATCGTATGTTC-3' and reverse 5'-GAAGTGTCTATCCTTATGAA-3'. The $25 \mathrm{~mL}$ PCR system consisted of $12.5 \mathrm{~mL}$ $2 \times$ SYBR Green master mix, $0.5 \mathrm{~mL}$ primer each, $1 \mathrm{~mL}$ cDNA tem-

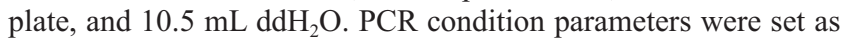
follows: $95^{\circ} \mathrm{C}$ for $10 \mathrm{~min} ; 95^{\circ} \mathrm{C}$ for $15 \mathrm{~s}$, and $60^{\circ} \mathrm{C}$ for $30 \mathrm{~s}$, for 40 45 cycles in total. Target gene expression levels were determined with the $2^{-\Delta \Delta \mathrm{Ct}}$ method. $\beta$-actin was used as an internal reference. 


\section{Western blot analysis}

Tissue (100 mg) was lysed with $500 \mathrm{~mL}$ RIPA lysis (containing $30 \mathrm{~mL}$ protease inhibitor) on ice. After centrifugation at $4^{\circ} \mathrm{C}$ at 12,000 rpm for $10 \mathrm{~min}$, the supernatant was collected. Sample protein concentration was determined with the BCA method (Beyotime, Haimen, Jiangsu, China). Totally $70 \mathrm{mg}$ protein sample was subjected to SDS-PAGE, and then electronically transferred onto the PVDF membrane. The membrane was blocked by $5 \%$ BSA at room temperature for $1 \mathrm{~h}$, and then incubated with rabbit-anti-rat anti-Bcl-2 polyclonal primary antibody (1:1000 dilution; Cell Signaling Technology, Beverly, CA, USA), rabbit-anti-rat anti-Caspase-3polyclonal primary antibody (1:1000 dilution; Cell Signaling), goat-anti-rat anti-IL-4 polyclonal primary antibody (1:1000 dilution; Santa Cruz, Santa Cruz, CA, USA), goat-anti-rat anti-IL-10 polyclonal primary antibody (1:1000 dilution; Santa Cruz), goat-anti-rat anti-TGFß1 polyclonal primary antibody (1:1000 dilution; Santa Cruz), goat-anti-rat anti-IFN- $\gamma$ polyclonal primary antibody (1:1000 dilution; Santa Cruz), rabbit-anti-rat anti-IL-2 polyclonal primary antibody (1:1000 dilution; Santa Cruz), goat-anti-rat anti-IL-6 polyclonal primary antibody (1:1000 dilution; Santa Cruz), rabbit-anti-rat anti-SDF-1polyclonal primary antibody (1:1000 dilution; Santa Cruz), goat-anti-rat anti-CXCR-4 polyclonal primary antibody (1:1000 dilution; Santa Cruz), rabbit-anti-rat anti-BDNF polyclonal primary antibody (1:1000 dilution; Santa Cruz), rabbit-anti-rat anti-erk1/2 polyclonal primary antibody (1:1000 dilution; Cell Signaling), rabbit-anti-rat anti-p-erk1/2 polyclonal primary antibody (1:1000 dilution; Cell Signaling), rabbit-anti-rat anti-trkB polyclonal primary antibody (1:1000 dilution; Cell Signaling), and rabbit-anti-rat anti-p-trkB polyclonal primary antibody (1:1000 dilution; Cell Signaling) respectively at $4{ }^{\circ} \mathrm{C}$ overnight. Then the membrane was incubated with corresponding secondary antibodies at room temperature for $2 \mathrm{~h}$. ECL colorization (Millipore) was performed, and protein band images were obtained and analyzed by the Image J software (ver. 1.44).

\section{Statistical analysis}

Data were expressed as mean \pm SD. SPSS 21.0 software was used for statistical analysis. The $t$-test and one-way ANOVA were performed for the group comparison, with the LSD- $t$ test for the pair-wise comparison. $\mathrm{P}<0.05$ was considered as statistically significant.

\section{Results}

\section{Effects of co-culture on subsets of monocytes and neu- ronal differentiation of BMSCs}

The effects of co-culture on the subsets of monocytes were first investigated. The proportion of CD4+ CD25+ T cells and the $\mathrm{CD} 4+/ \mathrm{CD} 8+$ ratio in the monocytes of co-culture 1 were analyzed by flow cytometry. Our results showed that, compared with the mononuclear transplantation group, the proportion of CD4+ CD25+ T cells $(5.13 \pm 0.36$ vs $10.97 \pm 0.24)$ and the CD4+/CD8+ ratio $(3.0 \pm 10.49$ vs $8.94 \pm 0.66)$ was significantly increased in the co-culture group $1(\mathrm{P}<0.05)$ (Figure 1).

Also, to investigate the effects of co-culture on SPIO-GFPBMSCs, the differentiation potential of these cells into neurons was analyzed with immunofluorescence staining. Herein, our results showed that, in the co-culture group 2, the proportions of $\mathrm{NeuN}^{+}$and NF-200 ${ }^{+}$cells were dramatically elevated compared to the BMSC transplantation group $(\mathrm{P}<0.05)$ (Figure 2). Taken together, these results suggest that, co-culture with SPIO-GFPBMSCs could significantly elevate the CD4+ CD25+ T cell proportion and $\mathrm{CD} 4+/ \mathrm{CD} 8+$ ratio in the monocytes, co-culture with monocytes could also significantly promote the neuronal differentiation of SPIO-GFP-BMSCs.

\section{Co-culture alters the protein contents in culture super- natant and in cell lysis buffer}

To further investigate the effects of co-culture on monocytes and SPIO-GFP-BMSCs, changes in the protein contents in the cell lysis buffer, as well as in the culture supernatant were analyzed by the protein array analysis. Our results showed that, compared with the SPIO-GFP-BMSCs cultured alone, the contents of vascular endothelial growth factor (VEGF), CD34, brain-derived neurotrophic factor (BDNF), and CXCR4 in cell lysis buffer were significantly elevated in both the co-culture groups 1 and 2 $(\mathrm{P}<0.001)$, and these protein contents in the co-culture group 2 were higher than in co-culture group $1(\mathrm{P}<0.05)$. On the other hand, in the culture supernatant, compared with the monocytes cultured alone, protein contents of VEGF, TGF- $\beta$, interleukin (IL)-4, and IL-10 were significantly elevated $(\mathrm{P}<0.05)$ (Tables 1

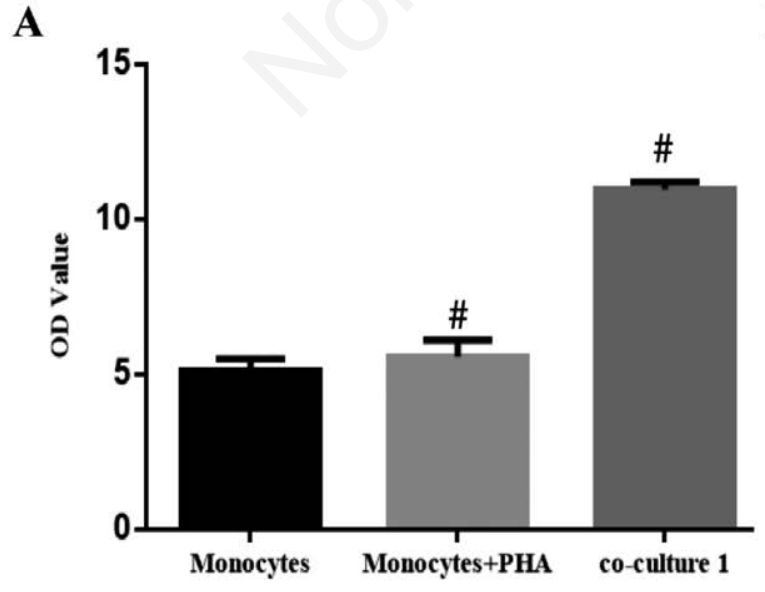

B

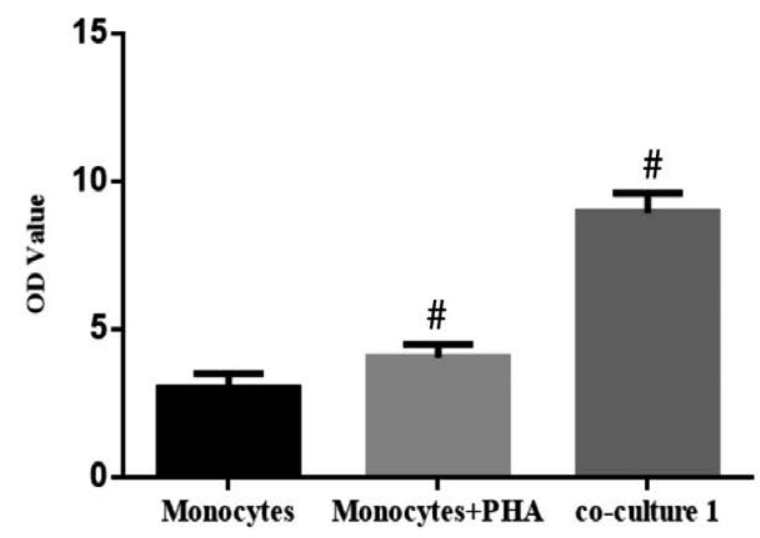

Figure 1. CD4+ CD25+ T cell proportion and CD4+/CD8+ ratio in monocytes co-cultured with BMSCs. Primary monocytes were cultured alone (with or without PHA) or co-cultured with SPIO-GFP-BMSCs, for $7 \mathrm{~d}$. Then monocyte subsets were detected with flow cytometry. The CD4+ CD25+ T cell proportion and CD4+/CD8+ ratio were obtained and analyzed accordingly. A) The CD4+ CD25+ T cell proportion. B) CD4+/CD8+ ratio was obtained and analyzed accordingly. Co-culture group 1, BMSCs:monocytes = 30:1. Error bars denote $\mathrm{SD}$. \#P<0.05, compared to the Monocytes group. 
and 2). The combination of these results suggests that, co-culture could affect the secretion of cytokines and, more precisely, promote the secretion of VEGF, BDNF, TGF- $\beta$, IL-4, and IL- 10 .
Co-transplantation decreases neuronal apoptosis in facial nerve nucleus in rats with FNA

TUNEL staining was performed in order to investigate the effects of co-transplantation of SPIO-GFP-BMSCs and monocytes on the neuronal apoptosis in the facial nerve nucleus of rats with FNA.

Table 1. Up-regulated protein levels in the cell lysis buffer.

\begin{tabular}{lccccc} 
& BMSCs & Co-culture 1 & Co-culture 2 & $F^{F}$ & \\
VEGF & $14599.5 \pm 123.56^{\mathrm{ab}}$ & $27269.67 \pm 98.65^{\mathrm{ac}}$ & $68460.07 \pm 79.91^{\mathrm{bc}}$ & 633.98 & 0.000 \\
CD34 & $349.25 \pm 22.32^{\mathrm{ab}}$ & $3330.14 \pm 59.56^{\mathrm{ac}}$ & $64471.63 \pm 32.47^{\mathrm{bc}}$ & 2819.56 & 0.001 \\
BDNF & $1435.5 \pm 52.65^{\mathrm{ab}}$ & $5370.67 \pm 75.63^{\mathrm{ac}}$ & $38736.96 \pm 83.34^{\mathrm{bc}}$ & 655.40 & 0.001 \\
CXCR4 & $1098 \pm 67.21^{\mathrm{ab}}$ & $908 \pm 42.18^{\mathrm{a}}$ & $4769.78 \pm 72.54^{\mathrm{bc}}$ & 285.17 & 0.001 \\
\hline
\end{tabular}

Compared with co-culture 2, a $\mathrm{P}<0.05$; compared with co-culture $1,{ }^{\mathrm{b}} \mathrm{P}<0.05$; compared with $\mathrm{BMSC}$, ${ }^{\mathrm{P}} \mathrm{P}<0.05$.

Table 2. Up-regulated protein levels in the co-culture supernatant.

\begin{tabular}{lccccc} 
& Monocytes & Co-culture 2 & Fold change & $t$ & $t$ \\
VEGF & $1227.96 \pm 108.34$ & $127746.60 \pm 82.13$ & 104.0315 & 1616.024 & 0.000 \\
TGF- $\beta$ & $663.17 \pm 13.17$ & $1850.24 \pm 49.61$ & 2.7927 & 40.557 & 0.000 \\
\hline IL-4 & $1232.00 \pm 56.12$ & $2833.60 \pm 81.65$ & 2.3083 & 28.171 & 0.000 \\
IL-10 & $1223.81 \pm 34.71$ & $4810.55 \pm 87.14$ & 3.9308 & 66.509 & 0.000 \\
\hline
\end{tabular}

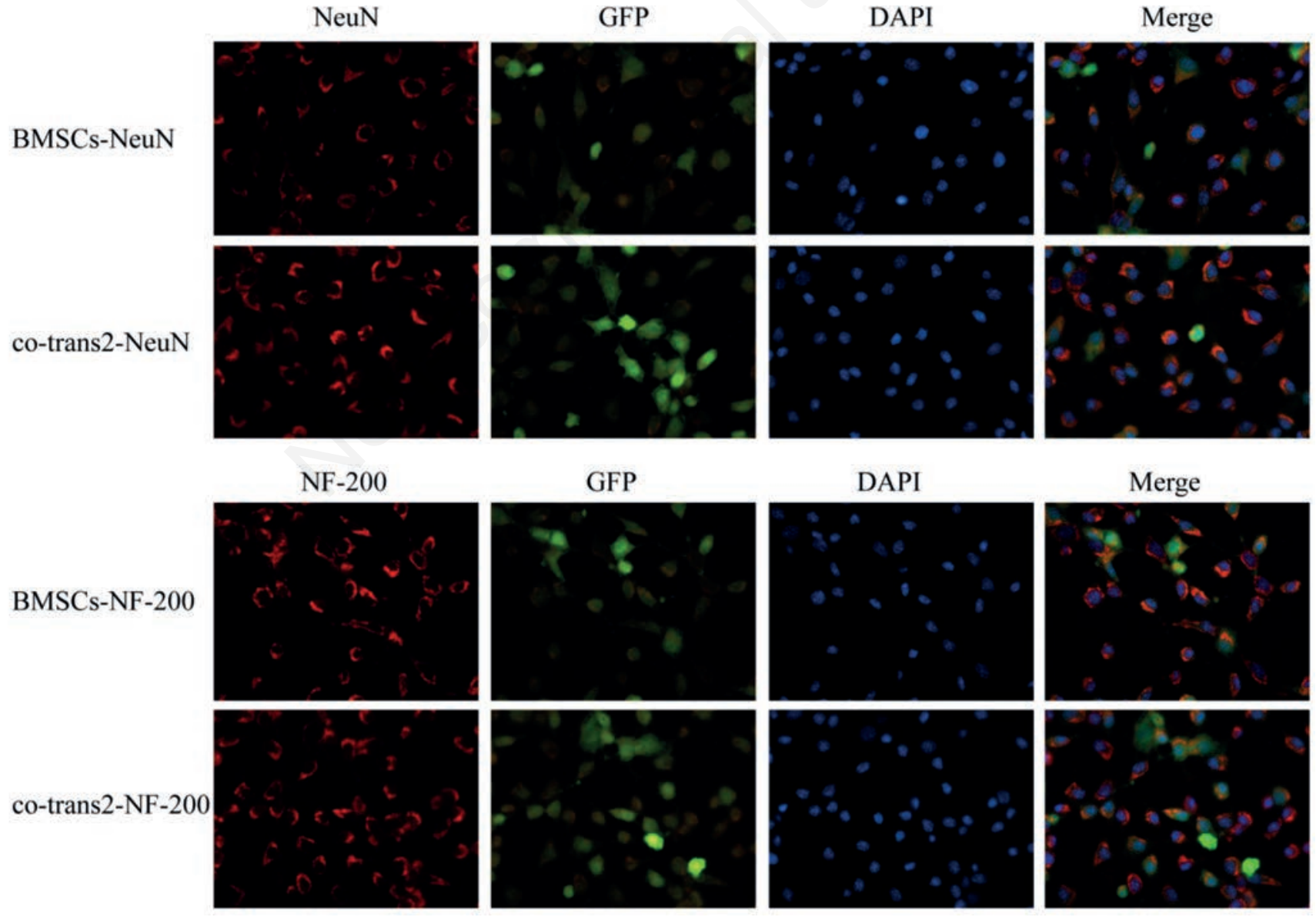

Figure 2. Effects of co-culture on the neuronal differentiation ability of BMSCs. SPIO-GFP-BMSCs were co-cultured with monocytes (at the ratio of 30:1) for $4 \mathrm{~d}$. Expression rates of NeuN and NF-200 were detected by immunofluorescence staining to assess the neuronal differentiation ability of BMSCs $\left({ }^{*} 200\right)$. Immunofluorescence of NeuN (red). Immunofluorescence of NF-200 (red). 
Our results showed that, in the NS group, the apoptotic neurons in the facial nerve nucleus rapidly increased from day 1 to day 14 after treatment, which then reached a platform from day 21 to day 28 after treatment. However, compared with the NS group, starting on day 7 after transplantation, the numbers of apoptotic neurons in the facial nerve nucleus dramatically declined in all the four cell transplantation groups $(\mathrm{P}<0.05)$. Among these cell transplantation groups, the apoptotic neurons mainly decreased in the co-transplantation group 2, followed by the co-transplantation group 1, while the neuronal apoptosis declined the least in the mononuclear cell transplantation group $(\mathrm{P}<0.05)$ (Figure $3 \mathrm{~A}, \mathrm{~B})$.

Furthermore, Western blot analysis showed that, in the NS group, the expression levels of apoptosis-related proteins, more specifically, Caspase-3 and Bcl-2 dramatically increased along with the time after FNA model establishment, which peaked on day 21 and then slightly declined.

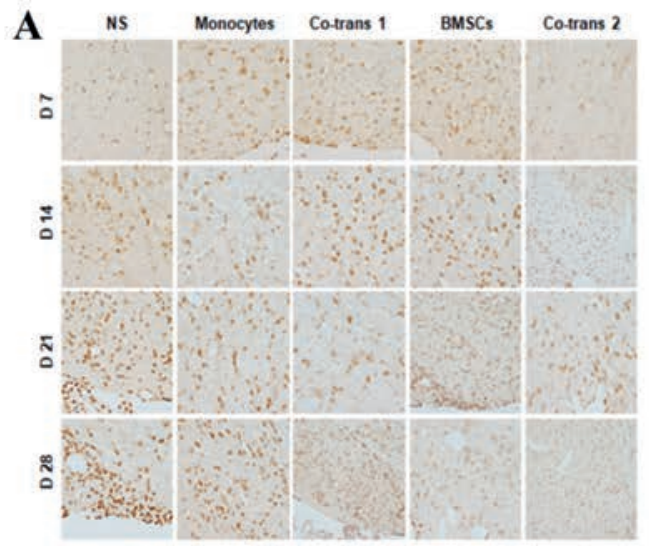

$\mathbf{B}$

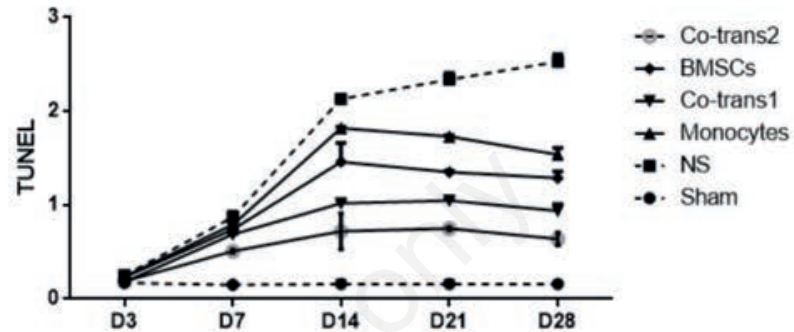

C
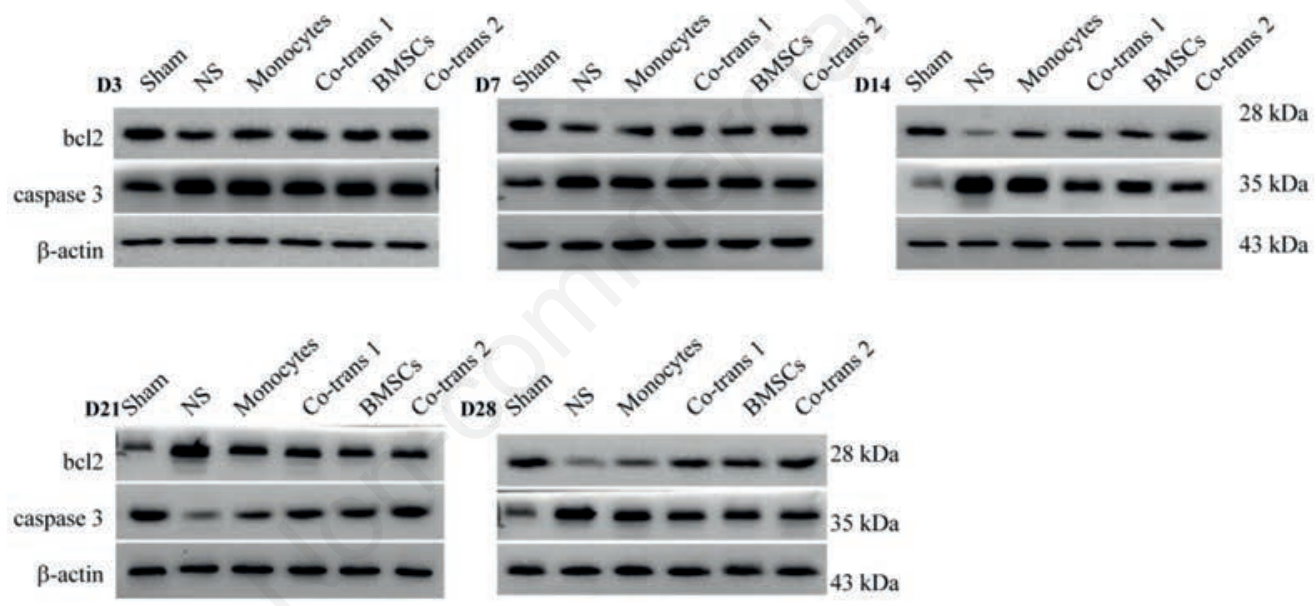

D

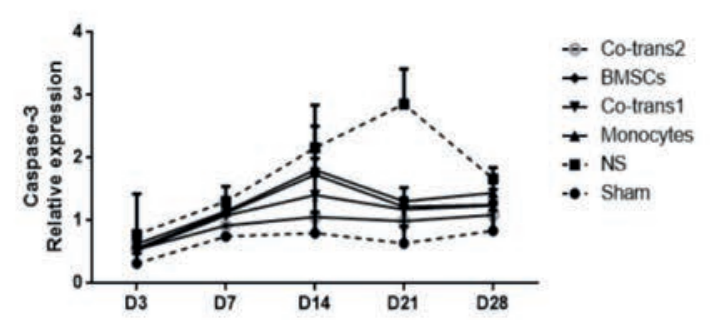

$\mathbf{E}$

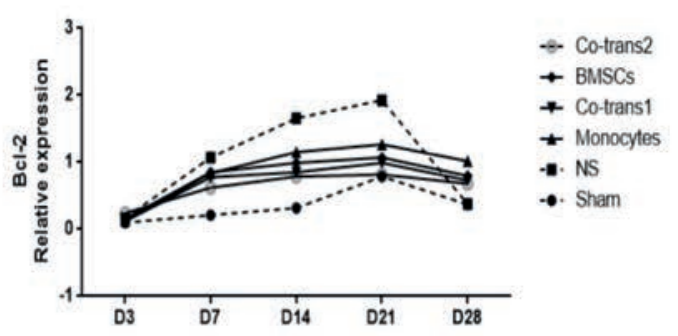

Figure 3. Neuronal apoptosis in the facial nerve nucleus after cell transplantation. A) FNA rats were transplanted with BMSCs and monocytes, either single culture or co-culture; the neuronal apoptosis in the facial nerve nucleus was detected with TUNEL staining, at indicated time points after cell transplantation. B) Statistical analysis of apoptotic neurons in the facial nerve nucleus. C) The expression levels of Caspase-3 and Bcl-2 in the facial nerve nucleus were detected with the Western blot analysis, at indicated time points after cell transplantation. D,E) Statistical analysis of Caspase-3 and Bcl-2 expression levels in the facial nerve nucleus. NS, positive control group; BMSCs, transplanted with SPIO-GFP-BMSCs alone; co-transplantation group 1, co-transplanted with SPIO-GFP-BMSCs and monocytes at the ratio of 30:1; monocytes, transplanted with monocytes alone; and co-transplantation group 2, co-transplanted with SPIO-GFP-BMSCs and monocytes at the ratio of 1:30. Error bars denote SD. 
Compared with the NS group, lower expression levels of Caspase- 3 were observed on day 3 after transplantation in four groups, which increased more significantly on day 21 but slightly declined on day 28 after cell transplantation $(\mathrm{P}<0.05)$ (Figure $3 \mathrm{C}$ E). The most obvious decrease of Caspase-3 proteins was observed on the co-transplantation group 2, followed by the co-transplantation group 1, while the mononuclear cell transplantation group owned the least difference. The expression of Bcl-2 also remained the lowest on the co-transplantation group 2, despite the significant decrease in the other group compared with $\mathrm{NC}$ group $(\mathrm{P}<0.05)$. The combination of these results suggested that co-transplantation of SPIO-GFP-BMSCs and monocytes could significantly reduce the neuronal apoptosis in facial nerve nucleus in rats with FNA.

A
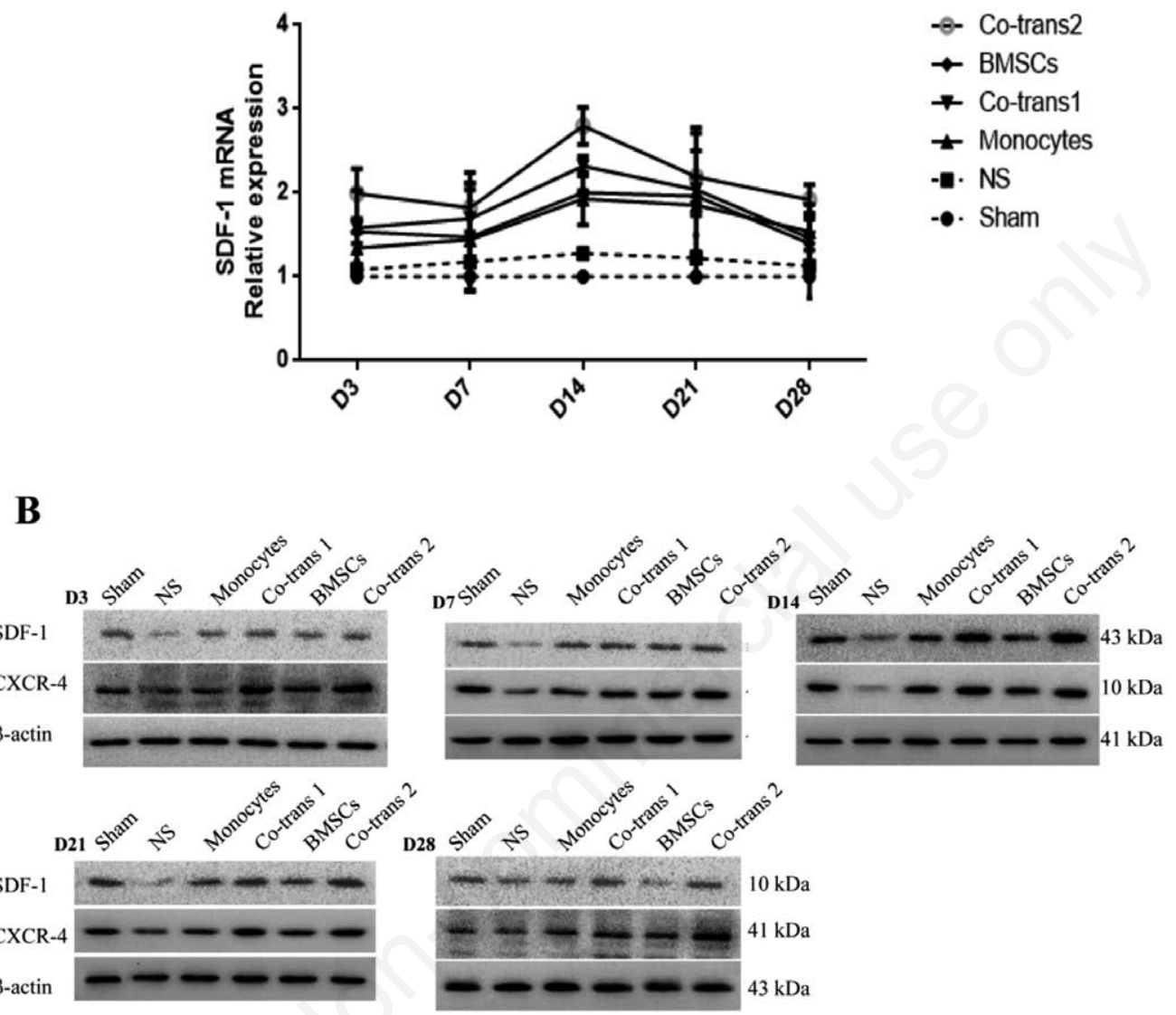

C

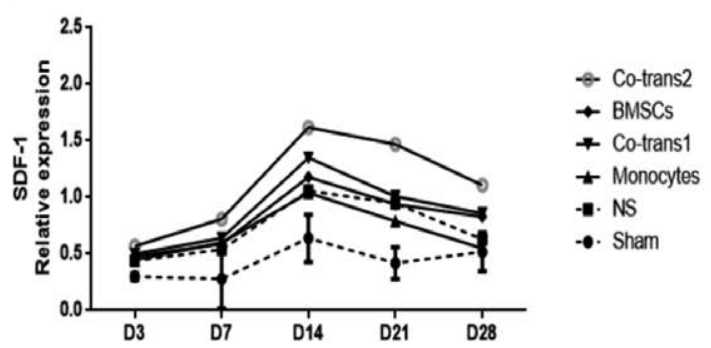

D

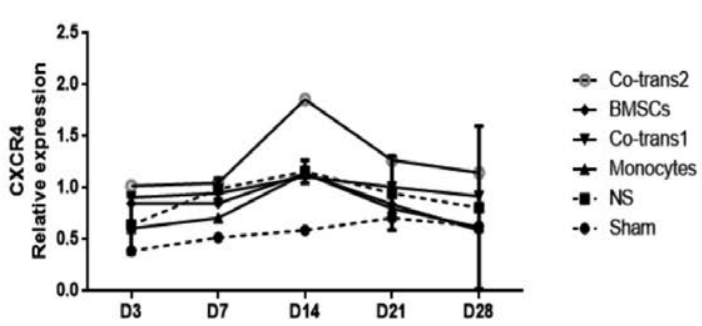

Figure 4. Expression levels of SDF-1/CXCR4 in the facial nerve nucleus after cell transplantation. A) Statistical analysis of SDF-1 mRNA expression levels in the facial nerve nucleus. B) FNA rats were transplanted with BMSCs and monocytes, either single culture or co-culture; the expression levels of SDF-1/CXCR4 in the facial nerve nucleus were detected with the Western blot analysis, at indicated time points after cell transplantation. C,D) Statistical analysis of SDF-1 and CXCR4 expression levels in the facial nerve nucleus. NS, positive control group; BMSCs, transplanted with SPIO-GFP-BMSCs alone; co-transplantation group 1, co-transplanted with SPIO-GFP-BMSCs and monocytes at the ratio of 30:1; monocytes, transplanted with monocytes alone; and co-transplantation group 2, co-transplanted with SPIO-GFP-BMSCs and monocytes at the ratio of 1:30. Error bars denote SD. 
Co-transplantation improves microenvironment for BMSC-neuron differentiation and growth in rats with FNA

We investigated the expression levels of chemokines (SDF$1 / C X C R 4)$ and neurotrophic factors in the facial nerve nucleus, to examine the effects of co-transplantation of SPIO-GFP-BMSCs and monocytes on the microenvironment for BMSC-neuron differentiation and growth. Our results from the quantitative real-time PCR showed that, compared with the control group, the SDF-1 mRNA expression levels were elevated in all the four cell transplantation groups, starting on day 3 after cell transplantation, peaking on day 14 , and slightly declining after that $(\mathrm{P}<0.05)$ (Figure $4 \mathrm{~A})$. The co-transplantation group 2 was significantly higher than all the other groups, followed by the co-transplantation group 1 . Moreover, for the NS group, the protein expression level of the SDF-1/CXCR4 axis started to increase on day 3 after treatment, which peaked on day 14. Compared with the NS group, the SDF$1 /$ CXCR4 expression levels were elevated in the four cell transplantation groups, which were all significantly higher than the sham group $(\mathrm{P}<0.05)$. The SDF-1/CXCR4 expression level in the co-transplantation group 2 was significantly higher than the other groups $(\mathrm{P}<0.05)$ (Figure $4 \mathrm{~B}-\mathrm{D})$.

Besides, the mRNA and protein expression levels of BDNF in the facial nerve nucleus were investigated. Our results showed that, in the NS group, the BDNF mRNA expression level was increased on day 3 after treatment, which peaked on day 14, and declined after that. Compared with the NS group, the BDNF mRNA expression levels in the four cell transplantation groups were significantly elevated on day 3 after transplantation, which peaked on day 14 (all significantly higher than the sham group), and declined after that (still higher than the NS group on day 28) $(\mathrm{P}<0.05)$. The highest BDNF mRNA expression level was observed in the co-transplantation group 2, followed by the cotransplantation group $1(\mathrm{P}<0.05)$ (Figure 5A). Similar results were observed in the detection of BDNF protein expression $(\mathrm{P}<0.05)$ (Figure 5 B,C). Since BDNF is generally recognized by experts in neural remodeling and function recovery, it is reasonable to indicate that co-transplantation of SPIO-GFP-BMSCs and monocytes affiliate chemotaxis homing of BMSC.

\section{Co-transplantation improves pro-inflammatory microenvironment in the facial nerve nucleus in rats with FNA}

To investigate the effects of co-transplantation of SPIO-GFPBMSCs and monocytes on the pro-inflammatory microenvironment in the facial nerve nucleus, the expression of pro-inflammatory cytokines in the facial nerve nucleus was detected. The protein expression levels of INF- $\gamma$, IL-2, and IL- 6 in the four cell transplantation groups were all lower than the NS group $(\mathrm{P}<0.05)$, but
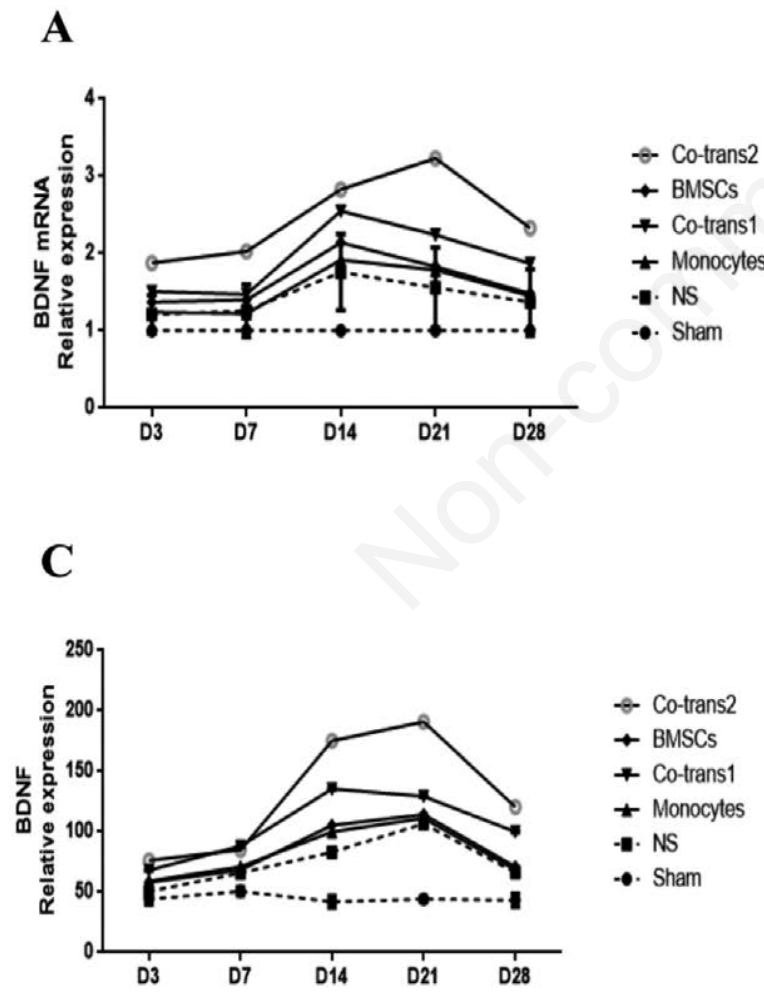

B

D7

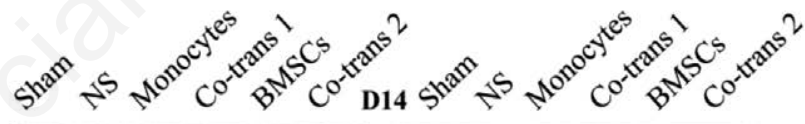

BDNF

trkB
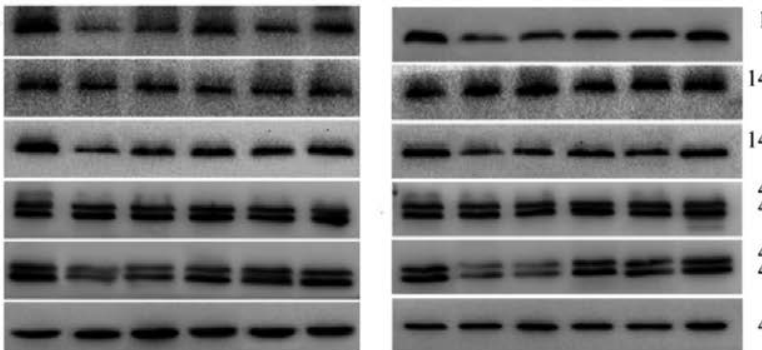

$14 \mathrm{kDa}$

$140 \mathrm{kDa}$

p-trkB

erk1/2

p-erk $1 / 2$

$\beta$-actin
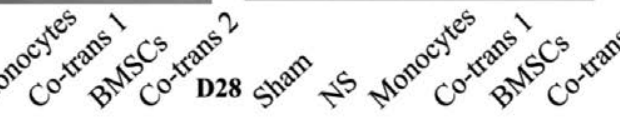

BDNF
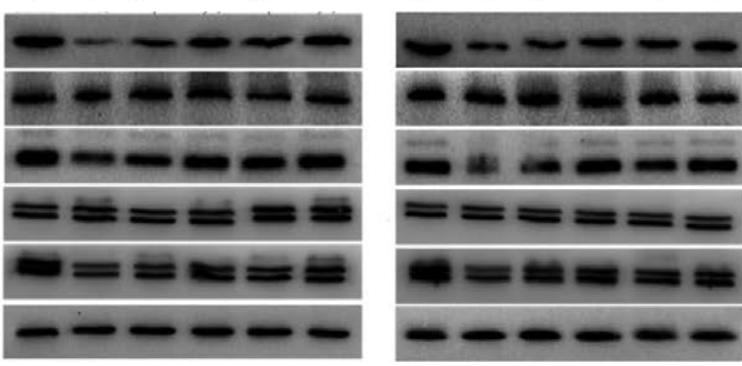

$14 \mathrm{kDa}$

$140 \mathrm{kDa}$

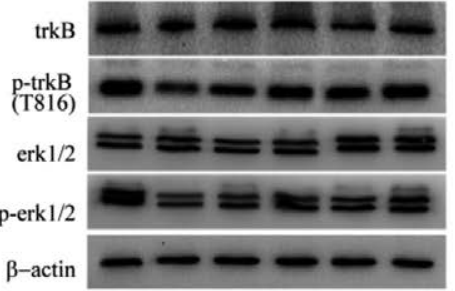

$140 \mathrm{kDa}$

${ }_{42}^{4} \mathrm{kDa}$

${ }_{42}^{4} \mathrm{kDa}$

$43 \mathrm{kDa}$

Figure 5. Expression levels of BDNF in the facial nerve nucleus after cell transplantation. A) Statistical analysis of BDNF mRNA expression levels in the facial nerve nucleus. B) FNA rats were transplanted with BMSCs and monocytes, either single culture or co-culture; the expression levels of BDNF in the facial nerve nucleus were detected with the Western blot analysis, at indicated time points after cell transplantation. C) Statistical analysis of BDNF expression levels in the facial nerve nucleus. NS, positive control group; BMSCs, transplanted with SPIO-GFP-BMSCs alone; co-transplantation group 1, co-transplanted with SPIO-GFP-BMSCs and monocytes at the ratio of 30:1; monocytes, transplanted with monocytes alone; and co-transplantation group 2, co-transplanted with SPIO-GFP-BMSCs and monocytes at the ratio of 1:30. Error bars denote SD. 
higher than the sham group $(\mathrm{P}<0.05)$. The pro-inflammatory cytokines decreased the most in the co-transplantation group 2, followed by the co-transplantation group 1, while declined the least in the mononuclear cell transplantation group $(\mathrm{P}<0.05)$ (Figure 6 $\mathrm{A}, \mathrm{B})$. Also, the mRNA and protein expression levels of antiinflammatory cytokines were analyzed by quantitative real-time PCR and western blot analysis. For the anti-inflammatory cytokines, quantitative real-time PCR showed that, except for the sham group, the IL-4 mRNA expression levels in the facial nerve nucleus were all elevated in the cell transplantation groups, starting on day 3 , peaking on day 14 or day 21 , and declining to normal levels on day 28 after transplantation $(\mathrm{P}<0.05)$ (Figure $7 \mathrm{~A}$ ). Moreover, western blot analysis showed that the protein expression levels of IL-4, IL-10 and TGF-1 were elevated on day 3 after treatment in all the groups, and then peaked on day 14 after transplantation and all slowly declined after that (still higher than the NS group), approaching to the expression of negative control on day $28(\mathrm{P}<0.05)$ (Figure $7 \mathrm{~B}-\mathrm{E})$. The anti-inflammatory cytokines increased the most in the co-transplantation group 2 . The combination of these results suggested that co-transplantation of SPIOGFP-BMSCs and monocytes could significantly decrease the proinflammatory cytokine expression and increase the anti-inflammatory cytokine expression, thus alleviating the pro-inflammatory microenvironment.

\section{Co-transplant repairs the FNA through the IL4/ JAK/STAT6 signaling pathway}

Furthermore, the analysis of the expression of proteins along the IL4/JAK/STAT6 signal pathway showed that, compared with the NS group, the expressions of JAK, p-JAK, signal transducer and activator of transcription 6 (STAT6), scos1 increased in all the four cell transplantation groups, starting on day 7 after transplantation, peaking on day 14 or day 21 , and declining after that, approaching to the negative control levels on day 28 . The most obvious changes were observed for the co-transplantation group 2, followed by the co-transplantation group 1, with less apparent changes in the single-cell transplantation groups $(\mathrm{P}<0.05)$ (Figure 8 A-D). Also, the neuronal apoptosis in the facial nerve nucleus in rats with FNA alleviates through the IL4/ JAK/STAT6 signaling pathways.

\section{Discussion}

Previous research showed that after cutting the spinal nerve root, it is possible to observe apoptosis and/or cell death could in several motor neurons of the spinal cord. ${ }^{12}$ Besides, apoptosis occurs not only in neurons missing axons but also in the long-dis-
$\mathbf{A}$

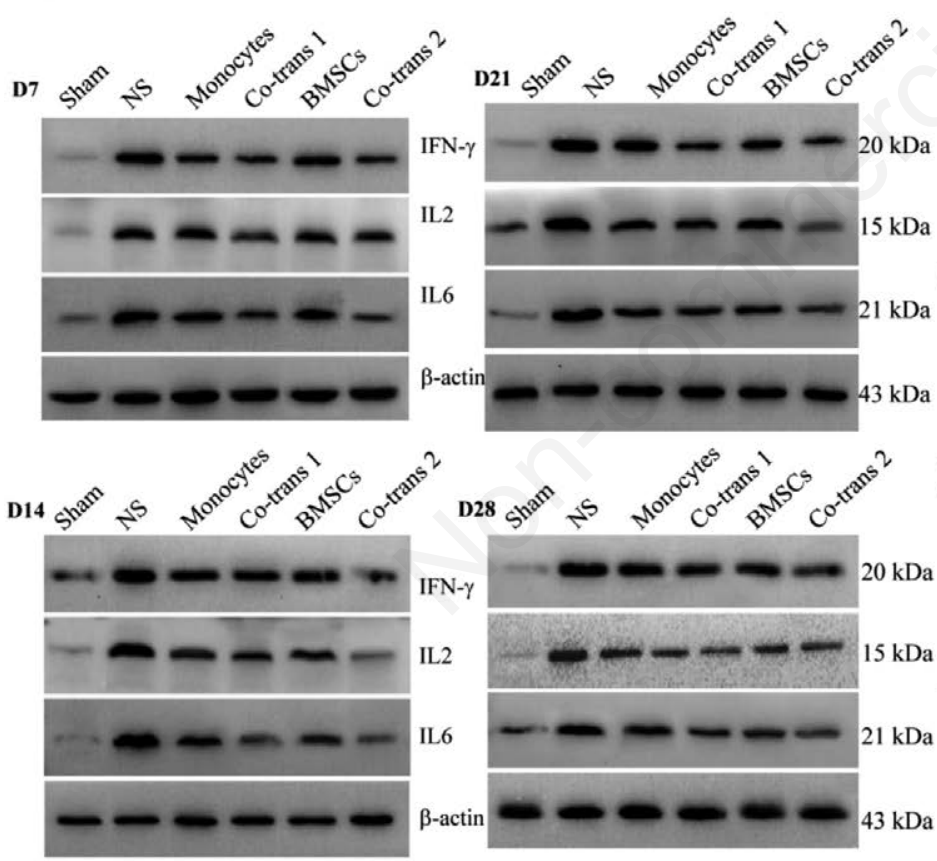

B
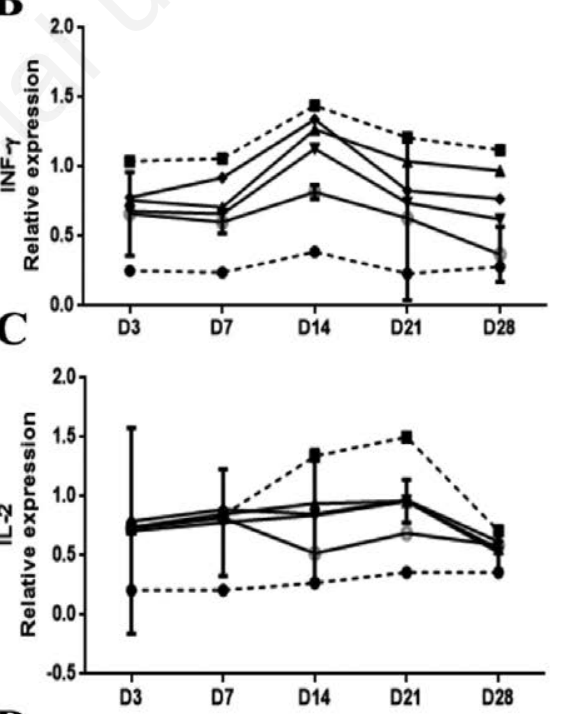

D

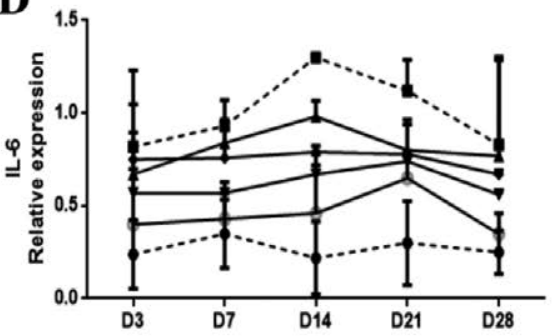

-8- Co-trans2

$\leftarrow$ BMSCs

$\rightarrow$ Co-trans1

+ Monocytes

- . NS

-. Sham

- Co-trans2

$\rightarrow$ BMSCS

* Co-trans1

- Monocytes

-n. NS

-• Sham

Figure 6. Expression levels of INF- $\gamma$, IL-2, and IL-6 in the facial nerve nucleus after cell transplantation. FNA rats were transplanted with BMSCs and monocytes, either single culture or co-culture. The expression levels of INF- $\gamma$, IL-2, and IL-6 in the facial nerve nucleus were detected with the western blot analysis, at indicated time points after cell transplantation. Statistical analysis of INF- $\gamma$, IL-2, and IL-6 expression levels in the facial nerve nucleus. NS, the positive control group; BMSCs, transplanted with SPIO-GFP-BMSCs alone; co-transplantation group 1, co-transplanted with SPIO-GFP-BMSCs and monocytes at the ratio of 30:1; monocytes, transplanted with monocytes alone; and co-transplantation group 2, co-transplanted with SPIO-GFP-BMSCs and monocytes at the ratio of 1:30. Error bars denote SD. 
tance neurons across the synapses, which might last for up to 6 months. In this study, the apoptotic processes in the brain stem of rat models of unilateral FNA were detected with the TUNEL staining at indicated time points after cell transplantation. Our results showed that the expression levels of Caspase-3 and Bcl-2 were significantly increased in the early period after injury, which peaked on day 21 and slowly declined after that. These results suggest that the cut of the facial nerve axon could induce apoptosis of FMNs in the facial nerve nucleus on the ipsilateral side, rather than on the contralateral side, which is in line with a recent study. ${ }^{13}$

SDF-1, together with the CXCR4 expressed on the surface of stem cells or lymphocytes, play an essential role in the regulation of the stem cells and inflammatory cells. Studies have shown that SDF-1/CXCR4 is the most important biological axis for the hom-

A

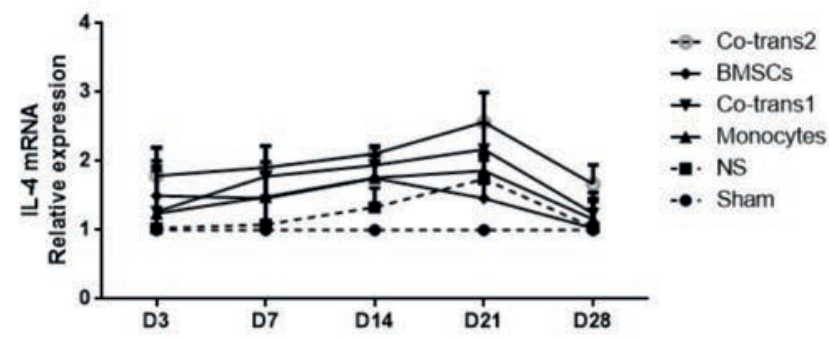

B

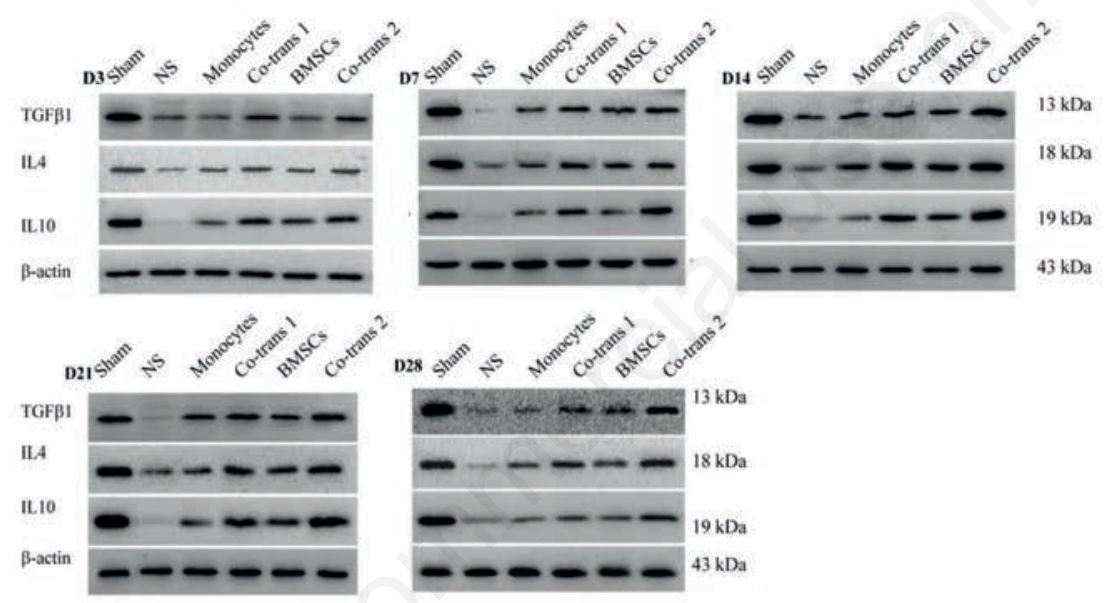

C
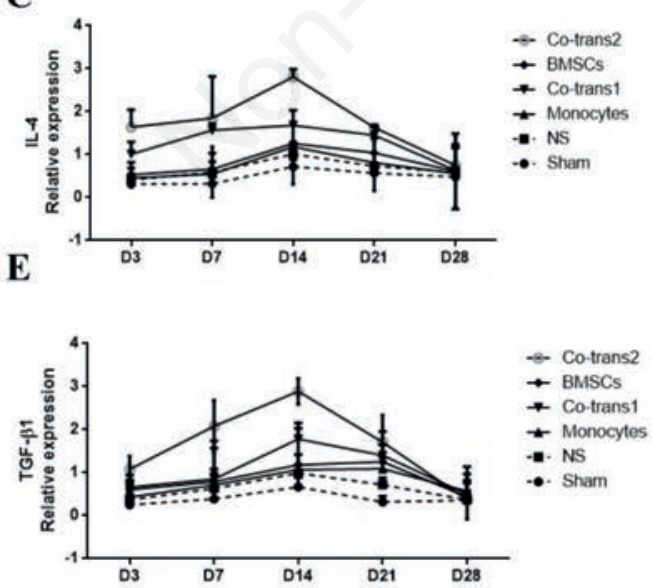

D

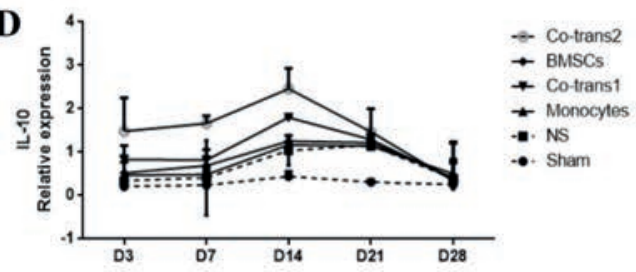

Figure 7. Expression levels of IL-4, IL-10, and TGF- $\beta 1$ in the facial nerve nucleus after cell transplantation. A) Statistical analysis of IL-4 mRNA expression levels in the facial nerve nucleus. B) FNA rats were transplanted with BMSCs and monocytes, either single culture or co-culture; the expression levels of IL-4, IL-10, and TGF- $\beta 1$ in the facial nerve nucleus were detected with the Western blot analysis, at indicated time points after cell transplantation. C-E) Statistical analysis of IL-4, IL-10, and TGF- $\beta 1$ expression levels in the facial nerve nucleus. NS, positive control group; BMSCs, transplanted with SPIO-GFP-BMSCs alone; co-transplantation group 1, cotransplanted with SPIO-GFP-BMSCs and monocytes at the ratio of 30:1; monocytes, transplanted with monocytes alone; and co-transplantation group 2, co-transplanted with SPIO-GFP-BMSCs and monocytes at the ratio of 1:30. $n=3$. Error bars denote SD. 
ing of transplanted BMSCs. The elevated expression of regulatory chemokine SDF-1 in the local injury region up-regulates the expression of CXCR4 in BMSCs, which makes the cells migrating to the lesion sites, inducing homing effects. ${ }^{14,15}$ Our results herein showed that except for the sham group, the expression of SDF$1 / \mathrm{CXCR} 4$ in the central facial nerve nucleus was significantly elevated after FNA, which peaked on day 14 after treatment and slowly declined after that. The changes could exert chemotaxis effects to recruit the inflammatory cells and could cause the movement of autologous stem cells towards the facial nerve nucleus. These results suggest that co-transplantation significantly elevates the expression of SDF-1/CXCR4 in the facial nerve nucleus, which recruits more BMSCs into it through the chemotaxis effects. This phenomenon plays a vital role in inhibiting the apoptotic processes of the facial neurons.

In this study, our results showed that after the model establishment, the anti-inflammatory cytokines (IL-4, IL-10, and TGF- $\beta 1$ ) were significantly elevated compared with the sham group, while the pro-inflammatory cytokines (INF- $\gamma$, IL-2, and IL-6) decreased. The facial neurons in the NS group suffered from obvious apoptosis, indicating pro-inflammatory microenvironment, which was not suitable for neuron survival. However, all the cell transplantation treatments reduced the amount of apoptotic facial neurons, and this might be attributed to the alleviated imbalance of the immune microenvironment in the facial nerve nucleus. Our results showed that, after the FNA, the expression of the JAK/STAT signal pathway had changed along with the expression of anti-inflammatory cytokine IL-4, which increased after injury immediately and peaked on day 14. It is possible to speculate about the fact that that IL-4 exerts its anti-inflammatory effect through the JAK/STAT signal pathway to regulate the immune microenvironment in the facial nerve nucleus. Also, signal transducer and activator of transcription (STAT) 4 and STAT6 are critical signals for the differentiation of CD4+ T cells into the CD4+ helper T cell 1 (Th1 cell) and CD4+ helper T cell 2 (Th2 cell) subgroups. ${ }^{17}$ In line with our results, Deboy et al. ${ }^{18}$ have shown that STAT6 could mediate $\mathrm{CH} 4+\mathrm{T}$ cells to differentiate into Th2 cells to secrete the antiinflammatory cytokines protecting the facial neurons.

In the co-culture, BMSCs inhibit the proliferation of CD8+ T cells, elevate the proportion of CD4 $+\mathrm{CD} 25+$ regulatory $\mathrm{T}$ cells, and increase the levels of anti-inflammatory cytokines IL-4, IL-10, and TGF- $\beta$ in the culture supernatant. BMSCs would benefit from the anti-inflammatory environment after transplantation. The elevated expression of CXCRC4 on the surface of BMSCs could promote the differentiation of BMSCs into the neuron-like cells or other neural cells in the microenvironment. These cells could replace the apoptotic neurons, or provide nutrition, finally reducing the neuronal apoptosis in the facial nerve nucleus. Moreover, our results showed that co-culture of BMSCs with monocytes (at the ratio of 1:30) could elevate the survival rate of facial neurons. Detection of proteins in the pro-inflammatory microenvironment in the facial nerve nucleus showed the highest IL-4, IL-10, and TGF- $\beta$ levels, lowest IL-2, and INF- $\gamma$ level, and the highest SDF1/CXCR4 and BDNF levels, in the co-transplantation group 2. Furthermore, the co-culture was associated with significantly elevated the proportion of CD4+ CD25+ T cells, which not only reg-
A
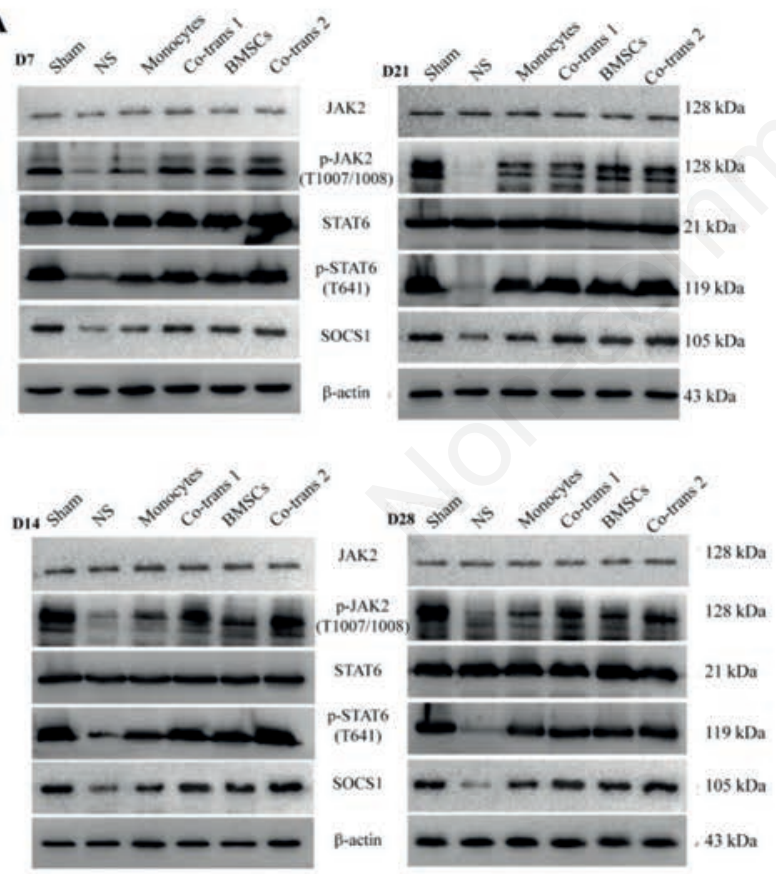
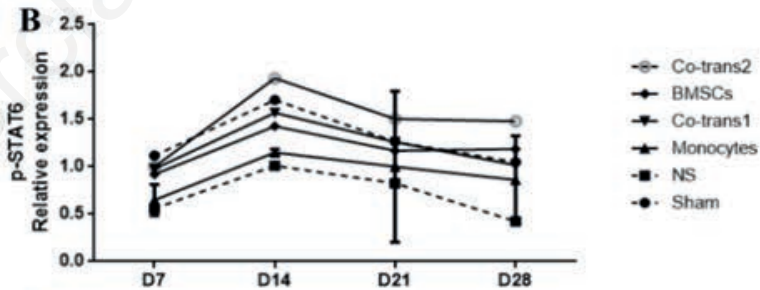

C
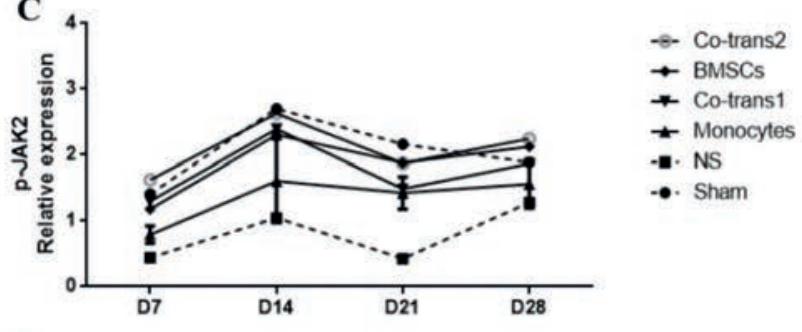

D

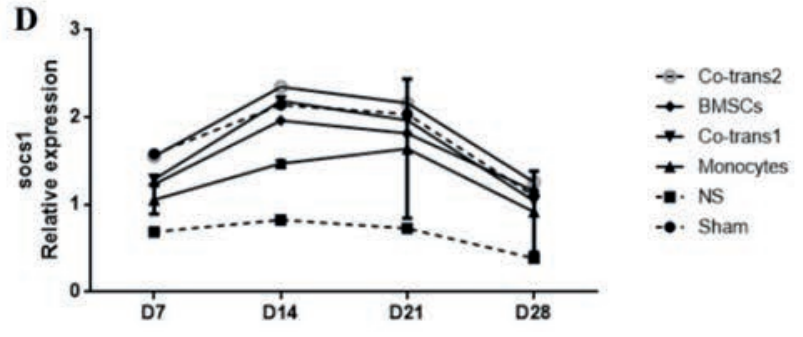

Figure 8. Expression levels of p-STAT6, p-JAK, and scos1 in the facial nerve nucleus after cell transplantation. A) FNA rats were transplanted with BMSCs and monocytes, either single culture or co-culture; the expression levels of p-STAT6, p-JAK, and scos1 in the facial nerve nucleus were detected with the Western blot analysis, at indicated time points after cell transplantation. B-D) Statistical analysis of p-STAT6, p-JAK, and scos1 expression levels in the facial nerve nucleus. NS, positive control group; BMSCs, transplanted with SPIOGFP-BMSCs alone; co-transplantation group 1, co-transplanted with SPIO-GFP-BMSCs and monocytes at the ratio of 30:1; monocytes, transplanted with monocytes alone; and co-transplantation group 2, co-transplanted with SPIO-GFP-BMSCs and monocytes at the ratio of $1: 30$. Error bars denote SD. 
ulate the microenvironment in the facial nerve nucleus but also repair the facial neurons themselves. The high neuronal survival rates in the co-transplantation groups might be due to the fact that condition is beneficial for its survival and differentiation, which also promotes the secretion of BDNF. After the FNA, inflammatory cytokines and/or neural growth factors might be produced in the facial nerve nucleus to activate the microglia, astrocytes, and peripheral immune cells around the FMN. The activated cells interact with the facial nerve neurons to change the gene expression in the nucleus, to provide the essential survival factors for the facial neurons, promoting the cell survival, axon regeneration, and fragment connection. ${ }^{19,20}$ The activated astrocytes could produce the neural growth factors, and activated microglia could secrete the excitatory neurotransmitters to deliver the signal to the motor nerve, thereby transforming the injured facial neuron synapses from the neurotransmitter transferring mode to the regenerative mode. ${ }^{20}$ Neuronal survival provides the foundation of functional repair after the FNA. BMSCs can differentiate into neuron-like cells or neural stem cells in the microenvironment in the central nervous system. Moreover, due to the local hypoxia or lack of blood flow, the BMSC survival rate is somehow decreased. Coculture of BMSCs and monocytes could inhibit the proliferation of monocytes, and increase the proportion of Treg cells, thereby improving the survival microenvironment for BMSCs and elevating the survival rate.

In conclusion, our results suggest that the co-transplantation of BMSCs and monocytes (BMSCs: monocytes $=1: 30$ ) could significantly improve the environment in the facial nerve nucleus, promoting the anti-/pro-inflammatory balance shift towards the antiinflammatory microenvironment, alleviating the survival conditions for BMSCs, regulating the chemotaxis, homing, differentiation, and section of BMSCs, and finally reducing the neuronal apoptosis. These findings might provide essential evidence for the in-hospital treatment of FNA with co-transplantation of BMSCs and monocytes.

\section{References}

1. Butler DP, Adriaan G. Facial palsy: what can the multidisciplinary team do? J Multidiscipl Healthc 2017;10:377-81.

2. Brazelton TR, Rossi FMV, Keshet GI, Blau HM. From marrow to brain: Expression of Neuronal phenotypes in adult mice. Science 2000;290:1775-9.

3. Mezey É, KJ Chandross, G Harta, RA Maki, SR McKercher. Turning Blood into Brain: Cells Bearing Neuronal Antigens Generated in Vivo from Bone Marrow. Science 2000;290:177982.

4. Melchiorri AJ, Nguyen BNB, Fisher JP. Mesenchymal stem cells: roles and relationships in vascularization. Tissue Eng Part B Rev 2014;20:218-28.

5. Casiraghi F, Azzollini N, Cassis P, Imberti B, Morigi M, Cugini D, et al. Pretransplant infusion of mesenchymal stem cells prolongs the survival of a semiallogeneic heart transplant through the generation of regulatory T cells. J Immunol 2008;181:3933-46.

6. Ge W, Jiang J, Baroja ML, Arp J, Zassoko R, Liu W, et al.
Infusion of mesenchymal stem cells and rapamycin synergize to attenuate alloimmune responses and promote cardiac allograft tolerance. Am J Transplant 2009;9:1760-72.

7. Nemeth K, Keane-Myers A, Brown JM, Metcalfe DD, Gorham $\mathrm{JD}$, Bundoc VG, et al. Bone marrow stromal cells use TGF- $\beta$ to suppress allergic responses in a mouse model of ragweedinduced asthma. Proc Natl Acad Sci USA 2010;107:5652-7.

8. Wang Y, Zhang A, Ye Z, Xie H, Zheng S. Bone marrow-derived mesenchymal stem cells inhibit acute rejection of rat liver allografts in association with regulatory T-cell expansion. Transplant Proc 2009;41:4352-6.

9. Ge W, Jiang J, Arp J, Liu W, Garcia B, Wang H. Regulatory Tcell generation and kidney allograft tolerance induced by mesenchymal stem cells associated with indoleamine 2,3-dioxygenase expression. Transplantation 2010;90:1312.

10. Kong QF, Sun B, Bai SS, Zhai DX, Wang GY, LiuYM, et al. Administration of bone marrow stromal cells ameliorates experimental autoimmune myasthenia gravis by altering the balance of Th1/Th2/Th17/Treg cell subsets through the secretion of TGFbeta. J Neuroimmunol 2009;207:83-91.

11. Dauer DJ, Huang Z, Ha GK, Kim J, Khosrowzadeh D, Petitto JM. Age and facial nerve axotomy-induced T cell trafficking: Relation to microglial and motor neuron status. Brain Behav Immun 2011;25:77-82.

12. Oliveira ALR. Apoptosis of sensory neurons and satellite cells after sciatic nerve transection in C57BL/6J mice. Bras J Med Biol Res 2001;34:375-380.

13. Hui L, Yuan J, Ren Z, Jiang X. Nerve growth factor reduces apoptotic cell death in rat facial motor neurons after facial nerve injury. Neurosciences 2015;20:65-8.

14. Hongbao L, Shuibing L, Yang L, Wang X, Xue W, Ge G, LuoX. The role of SDF-1-CXCR4/CXCR7 axis in the therapeutic effects of hypoxia-preconditioned mesenchymal stem cells for renal ischemia/reperfusion injury. Plos One 2012;7:e34608.

15. López Ponte A, Marais E, Gallay N, Langonné A, Delorme B, Hérault $\mathrm{O}$, et al. The in vitro migration capacity of human bone marrow mesenchymal stem cells: comparison of chemokine and growth factor chemotactic activities. Stem Cells 2007;25:173745.

16. Xin J, Wainwright DA, Serpe CJ, Sanders VM, Jones KJ. Phenotype of CD4+ T cell subsets that develop following mouse facial nerve axotomy. Brain Behav Immun 2008;22:528-37.

17. Deboy CA, Xin J, Byram SC, Serpe CJ, Sanders VM, Jones KJ. Immune-mediated neuroprotection of axotomized mouse facial motoneurons is dependent on the IL-4/STAT6 signaling pathway in CD4+ T cells. Exp Neurol 2006;201:212-24.

18. Jones KJ, Serpe CJ, Byram SC, Deboy CA, Sanders VM. Role of the immune system in the maintenance of mouse facial motoneuron viability after nerve injury. Brain Behav Immun 2005;19:019.

19. Sanders VM, Jones KJ. Role of immunity in recovery from a peripheral nerve injury. J Neuroimm Pharmacol 2006;1:11-9.

20. Oliveira ALR, Thams S, Lidman O, Piehl F, Hökfelt T, Kärre K, et al. A role for MHC class I molecules in synaptic plasticity and regeneration of neurons after axotomy. Proc Natl Acad Sci USA 2004;101:17843-8.

Received for publication: 28 March 2020. Accepted for publication: 13 May 2020.

This work is licensed under a Creative Commons Attribution-NonCommercial 4.0 International License (CC BY-NC 4.0).

(C) Copyright: the Author(s), 2020

Licensee PAGEPress, Italy

European Journal of Histochemistry 2020; 64(s2):3136

doi:10.4081/ejh.2020.3136 